\title{
Motion of Euglena Gracilis: Active Fluctuations and Velocity Distribution
}

\author{
P. Romanczuk · M. Romensky · D. Scholz • \\ V. Lobaskin • L. Schimansky-Geier
}

the date of receipt and acceptance should be inserted later

\begin{abstract}
We study the velocity distribution of unicellular swimming algae Euglena gracilis using optical microscopy and theory. To characterize a peculiar feature of the experimentally observed distribution at small velocities we use the concept of active fluctuations, which was recently proposed for the description of stochastically self-propelled particles [Romanczuk, P. and Schimansky-Geier, L., Phys. Rev. Lett. 106, 230601 (2011)]. In this concept, the fluctuating forces arise due to internal random performance of the propulsive motor. The fluctuating forces are directed in parallel to the heading direction, in which the propulsion acts. In the theory, we introduce the active motion via the depot model [Schweitzer et al., Phys. Rev. Lett. 80, 23, 5044 (1998)]. We demonstrate that the theoretical predictions based on the depot model with active fluctuations are consistent with the experimentally observed velocity distributions. In addition to the model with additive active noise, we obtain theoretical results for a constant propulsion with multiplicative noise.
\end{abstract}

P. Romanczuk

Department of Ecology and Evolutionary Biology, Princeton University, Princeton, New Jersey 08544, USA; Thaer-Institute, Humboldt Universität zu Berlin, 10099 Berlin

M. Romensky

Department of Mathematics, Uppsala University, Box 480, Uppsala 75106, Sweden

D. Scholz

Conway Institute, University College Dublin, Belfield, Dublin 4, Ireland

V. Lobaskin

School of Physics, Complex and Adaptive Systems Lab, University College Dublin, Belfield, Dublin 4, Ireland,

E-mail: vladimir.lobaskin@ucd.ie

L. Schimansky-Geier

Department of Physics, Humboldt Universität zu Berlin, Newtonstr. 15, 12489 Berlin, Germany,

E-mail: alsg@physik.hu-berlin.de 


\section{Introduction}

The development of the theory of Brownian motion, connected to such famous names as Einstein [1, Langevin [2] or Smoluchowski [3, was one of the greatest triumphs in statistical physics at the beginning of the $20^{\text {th }}$ century. Since then Brownian motion not only assumed a central role in the foundation of thermodynamics and statistical physics but continues to date to be a major interdisciplinary research topic.

Recently there has been an increasing focus in biology, physics and chemistry, on so-called active matter systems, both from experimental and theoretical point of view. Examples of such far-from-equilibrium systems range from the dynamical behavior of individual units such as Brownian motors [4, motile cells [5-10, macroscopic animals [11 13] or artificial self-propelled particles [14 18] to large coupled ensembles of such units and their large scale collective dynamics 19 26]. Major advances in active matter research have been made possible by the continuously improving experimental techniques such as for example automated digital tracking [7, 8, 13, 23] or the realization of active granular and colloidal systems [14, 15, 18, 27.

Despite these recent advances, there are still many open questions regarding the universal properties of active motion. Already at the level of individual active particles it is important to distinguish thermal fluctuations resulting from the interactions with a heat bath from effective stochastic forces which have their origin in the active nature of the system. Recently, it was shown that such active fluctuations may lead to a complex behavior of the mean squared displacement of self-propelled particles with multiple crossovers [25, 28, and may manifest themselves in characteristic behavior of probability density functions at small speeds, strikingly different from the ones obtained for thermal fluctuations [25, 29]. Nontrivial motion statistics and patterns also arise in systems that can switch between two or more locomotion modes like run-and-tumble [30, 31] or two different speed states 31,32 .

We should note that the term "active fluctuations" has been used before to refer to non-equilibrium fluctuations of intracellular filament networks (see e.g. [33 35]), and recently also in the context of flagellar beating [36]. Although all these concepts can be related on a very fundamental level, here we restrict our definition of active fluctuations to the velocity fluctuations of self-propelled agents, which can be described as active Brownian particles [25.

In this work we report on experimental observed velocity distribution of unicellular swimming algae Euglena gracilis, which cannot be explained by models with passive noise only. To describe the motion of the cells, we develop a theory of active Brownian motion with active fluctuations and derive the corresponding particle velocity distributions. We demonstrate that the measured velocities are consistent with the theoretical predictions, which allows for insight into the motion characteristics and control patterns.

The remainder of the paper is organized as follows: In Section 2 we describe the experimental setup used to measure velocity distributions of Euglena gracilis. Further on, in Section 3, we introduce the theory and discuss the comparison between the theory and experiment. In addition, we discuss also a second theoretical model, to demonstrate that multiplicative noise can be also considered within the active fluctuations framework. Finally, we conclude in Section 4. 
2 Experiment

\subsection{System}

In this work, we studied the motion of unicellular photosynthetic flagellate Euglena gracilis Klebs using optical microscopy. It is known that Euglena gracilis is most sensitive to blue light: the photosynthetic efficiency is maximal for $465 \mathrm{~nm}$ light [37. Euglena is a flagellated swimmer (a pusher-type), propelled by a single flagellum. The motion is not purely phototactic: Euglena cells in dark conditions perform random motion with much spinning [38. The threshold intensity of blue light required to cause the negative phototaxis is approximately $10 \mathrm{~mW} / \mathrm{cm}^{2}[39$. This is an almost two times lower limiting light intensity than that for a normal daylight [40]. In our experiments we investigated behavior of the cells in strong blue light beyond the photosynthetic limit when the photophobic behavioural reactions are most pronounced. The cells were supplied by Carolina Biological Supply Company (Burlington, NC, USA) and grown on an aerated, inorganic salt medium at room temperature under a 12:12 hours light-dark cycle. The average cell size (length) was about $40 \mu \mathrm{m}$.

The schematic of the experimental setup is shown in Fig. 1 During each experimental trial the cells were confined inside a microfluidic channel (50 mm length, $5 \mathrm{~mm}$ width, $400 \mu \mathrm{m}$ height and $100 \mu \mathrm{l}$ volume) of an ibiTreat 0.4 Luer channel slide installed into a slide-mount on the stage of the inverted microscope. Small height of the channel allowed us to consider motion in two spatial dimensions. The tubes on both ends of the channel were kept open during the experiment ensuring proper gaseous cell metabolism. Illumination of the cells with light was performed by two light emitting diodes (LED) positioned in the central part of the channel so that the angle between the longitudinal axis of each LED and the channel plane was fixed at $60^{\circ}$ and the distance between the centers of diodes was $10 \mathrm{~mm}$. This arrangement takes into account characteristics of the directivity pattern of each LED and ensures uniformity of the illumination within the view field of the optical system. In this work, we used a pair of blue LEDs with a peak emission at $465 \mathrm{~nm}$ (NSPB500S, Nichia Corporation , Japan). The diodes were lighted up simultaneously. The light intensity was set to $50 \mathrm{~mW} / \mathrm{cm}^{2}$ - well beyond the photosynthetic limit. The activation light from the diodes has been used for detection, no additional illumination was required. All experiments were performed at room temperature. Duration of each run was about 10 minutes.

\subsection{Imaging and data collection}

The optical readout system consisted of an image acquisition equipment and data processing computer. Real time images of Euglena cells in a channel slide were captured by the Andor iXon3 885 EMCCD camera $(1004 \times 1004$ pixels, pixel size $8 \mu \mathrm{m}, 2$ fps frame rate) mounted on the optical microscope Zeiss Axiovert $200 \mathrm{M}$ through a $5 \mathrm{x}$ objective lens. The observation field was $1.6 \mathrm{~mm} \times 1.6 \mathrm{~mm}$. The number of cells within the observation area was $800-1200$ giving packing fractions of $0.026-0.039$. Images were recorded in miltipage TIFF format using original camera manufacturer software. Typical trajectories of the cells are shown in Fig. 2 . 


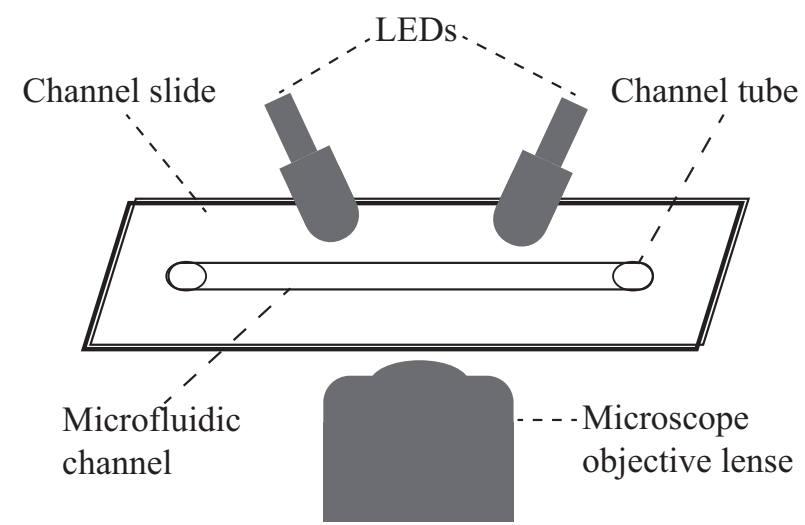

Fig. 1 Scheme of the experimental setup.



Fig. 2 Typical trajectories of Euglena gracilis cells captured in strong blue light. Only ten trajectories are shown for the sake of clarity.

Further image processing involving cell recognition and tracking was performed with Imaris package, v7.6 (Bitplane, UK). The raw data consisted of $x$ and $y$ coordinates of the cell geometrical centers, identities of cells and a time stamp. These data did not include positions of non-motile (dead) cells which were excluded with a filter based on a minimal trajectory length $(50 \mu \mathrm{m})$. In the videos, the frame rate seems to be sufficiently high. The displacements between the frames are less than the cell size. The positional statistics was subsequently exported into ASCII format files and analyzed with our code. Velocities of the cells were calculated based on the two subsequent positions. The motion statistics were averaged over two runs. Due to isotropy of the motion, we combined the histograms in $x$ and $y$ directions. To extract the slice of the histogram corresponding to one velocity component $\left(v_{x}, 0\right)$, we used the data where the corresponding transverse component $v_{y}$ was small $-0.5 \mu \mathrm{m} / \mathrm{s}<v_{y}<0.5 \mu \mathrm{m} / \mathrm{s}$.

The cells in strong blue light were in constant motion, although slowing down from time to time. As we noticed the change of the motion within the duration of the experiment, we split the statistics into 6 subsets corresponding to time 

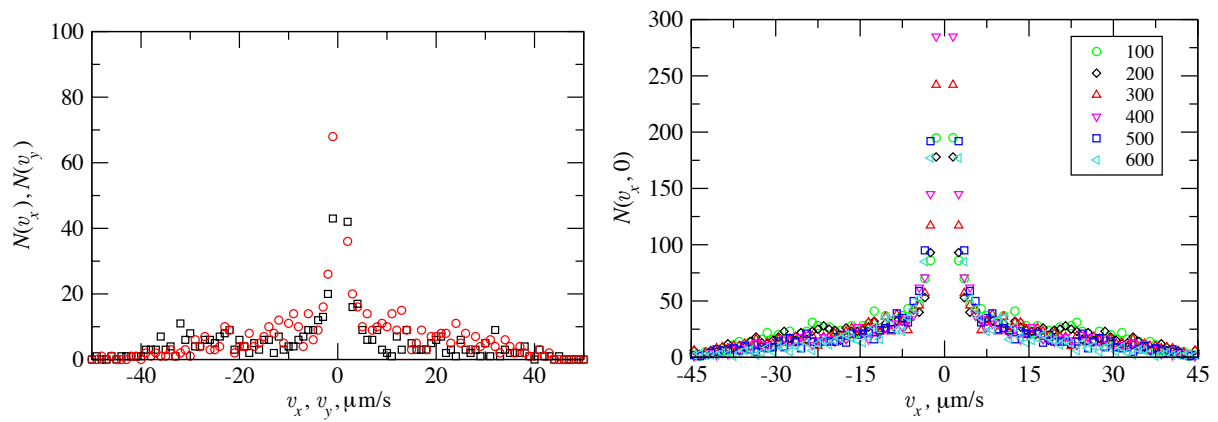

Fig. 3 (a) An example of raw data set for Euglena gracilis in strong blue light collected 200300 seconds from the start of the illumination. (b) Six symmetrised datasets, where $\pm v_{x}$ and $\pm v_{y}$ are combined.

intervals of 0-100, 100-200, 200-300, 300-400, 400-500, and 500-600 seconds. An example of a raw dataset with $v_{x}$ and $v_{y}$ velocity components for one interval and the collection of symmetrised data are shown in Fig. 3. We can observe straight away a striking feature of these distributions: A strong peak around zero velocity. This is in contrast with the shape of distribution predicted for active Brownian particles effected by thermal agitation, likewise usual Brownian motion which have a crater like shape with a minimum around zero. This agitation from the molecules of the surrounding liquid is negligible compared to the phototactic propulsion. To understand the peak at vanishing velocity we need to introduce a different noise model, which we present in the next section.

\section{Self propelled particles with active fluctuations}

\subsection{Models with active fluctuations}

In recent publications two of us have investigated the difference between passive and active noise acting on the motion of self propelled units [25, 29]. Passive fluctuations have their origin in the fluctuating environment in which the particle moves. In result, the effect of a passive random force $\boldsymbol{\eta}_{\mathrm{p}}(t)$ is independent on the direction of motion (heading) of the particle. The classical example of passive fluctuations is ordinary Brownian motion in thermal equilibrium, where the stochastic force is associated with random collisions of the Brownian particle with molecules of the surrounding fluid.

In contrast, active fluctuations $\boldsymbol{\eta}_{\mathrm{a}}(t)$ are a pure far from equilibrium phenomenon. They may become important in the motion of an biological agents or self-propelled particles. The origin of these fluctuations can be for example variations in the propulsion of chemically powered colloids 14 16, complex intracellular processes in cell motility [7, 8] or unresolved internal decision processes in animals [11 13 .

In this manuscript we show that the concept of active fluctuations is able to explain measurements of the velocity distribution function of the Euglena cells. Especially, an observed giant peak in the two dimensional velocity distribution of the Cartesian velocity components $P\left(v_{x}, v_{y}\right)$ at the origin $v_{y}=0, v_{y}=0$ is due to 
the special selection of the noise sources. Only active fluctuations describe correctly the large peak in two-dimensional Cartesian velocity distributions at small speeds.

We assume a two-dimensional motion of the cells is given by the position vector $\boldsymbol{r}(t)$ and the velocity $\boldsymbol{v}(t)$. Components are labeled as $\mathbf{r}=(x, y)$ for the position vector of the cell, and $\mathbf{v}=\left(v_{x}, v_{y}\right)$ being its velocity vector. The dynamics of the kinematics of the cell is given by the second Newton law. We assume a propulsive mechanism with force $\boldsymbol{a}_{p}$, Stokes friction and a random forces $\boldsymbol{\eta}(t)$ acting on the cell. In particular, the dynamics reads afterwards.

$$
\begin{aligned}
\dot{\mathbf{r}} & =\mathbf{v} \\
m \dot{\mathbf{v}} & =\boldsymbol{a}_{p}-\gamma \mathbf{v}+\boldsymbol{\eta}(t)
\end{aligned}
$$

and we will set the mass to unity $m=1$, later on.

Active particles have a preferred direction of motion (heading) determined by their propulsion mechanism ("head-tail" asymmetry). The heading vector is a unit vector $\mathbf{e}_{h}$ with $\left|\mathbf{e}_{h}\right|=1$ defining the orientation of the particle along which the propulsive force acts. This force is independently on other acting external forces. It acts generally in direction of the instantaneous velocity, and can be decelerating or accelerating.

In two spatial dimensions $(d=2)$ it is fully determined by the angle $\varphi$ defining the direction with respect to the $x$-axis (see Fig. 4a): $\mathbf{e}_{h}(t)=(\cos \varphi(t), \sin \varphi(t))$, and the evolution of the position of the particle can be rewritten as

$$
\dot{\mathbf{r}}(t)=\mathbf{v}(t)=v(t) \mathbf{e}_{h}(t) .
$$

Note, that $v(t)$, which is the projection of the velocity onto the heading direction, can also adopt negative values if moving backwards with respect to the propulsive mechanism. The heading vector differs from the unit vector of the velocity $\mathbf{e}_{v}(t)$ to which it is either parallel or anti-parallel if moving forward or backward. Projected on $\mathbf{e}_{v}(t)$, the velocity is the speed and always positive and the length of the vector $\mathbf{v}(t)=s(t) \mathbf{e}_{v}(t)$ with $s(t)=\sqrt{v_{x}^{2}+v_{y}^{2}}=|\boldsymbol{v}(t)|$.

The temporal evolution of the velocity vector in the new coordinates $(v, \varphi)$ reads

$$
\dot{\mathbf{v}}=\dot{v} \mathbf{e}_{h}+v \dot{\varphi} \mathbf{e}_{\varphi}
$$

where $\mathbf{e}_{\varphi}=(-\sin \varphi(t), \cos \varphi(t))$ is the unit vector in the angular direction. Multiplying Eq. (2) with $\mathbf{e}_{v}$ and $\mathbf{e}_{\varphi}$, respectively, yields:

$$
\begin{aligned}
& \dot{v}=a_{p}-\gamma v+\boldsymbol{\eta} \cdot \boldsymbol{e}_{h}, \\
& \dot{\varphi}=\frac{1}{v} \boldsymbol{\eta} \cdot \boldsymbol{e}_{\varphi} .
\end{aligned}
$$

Here, - indicates a scalar product. One has to note that the orientational dynamics diverge for $v=0$. This is due to the fact that for a point-like particle, as considered here, the polar angle $\varphi$ cannot be defined for a vanishing speed.

At this point one has to distinguish between the action of active vs passive noise. Passive noise as in case of Brownian motion acts independently in the $x$ and $y$ directions $\boldsymbol{e}_{x}$, respectively $\boldsymbol{e}_{y}$, as Gaussian white noise. It decomposes into two independent components with

$$
\boldsymbol{\eta}_{\mathrm{p}}(t)=\sqrt{2 D}\left(\xi_{x}(t) \boldsymbol{e}_{x}+\xi_{y}(t) \boldsymbol{e}_{y}\right)
$$



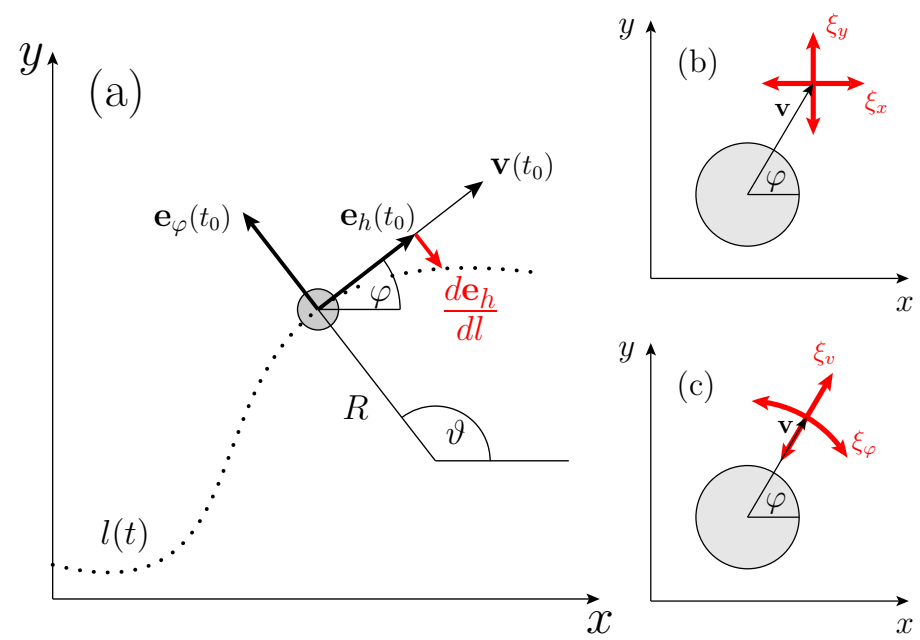

Fig. 4 (a) Schematic visualization of motion in a plane with unit vectors $\boldsymbol{e}_{h}(t), \boldsymbol{e}_{\varphi}(t)$ (thick arrows), velocity vector $\boldsymbol{v}(t)$ (thin arrow) and $\mathrm{d} \boldsymbol{e}_{h} / \mathrm{d} l$ (thick red/gray arrow). The trajectory of the particle $l(t)$ is indicated by the dotted line. (b) Visualization of passive fluctuations $\xi_{x}(t)$ and $\xi_{y}(t)$ (thick red/gray arrows). (c) Visualization of active fluctuation $\xi_{v}(t)$ and $\xi_{\varphi}(t)$ (thick red/gray arrows). Figure reproduced from [49.

with following properties

$$
\left\langle\xi_{i}(t)\right\rangle=0, \quad\left\langle\xi_{i}(t) \xi_{j}\left(t^{\prime}\right)\right\rangle=\delta_{i, j} \delta\left(t-t^{\prime}\right), \quad i, j \in\{x, y\}
$$

Thus with this choice of velocity variables, the problem becomes a model with multiplicative noise in (5). It is easier solved in a Cartesian representation of the velocity vectors, where for passive noise the motion in $x$ and $y$ becomes independent.

In contrast to passive noise, for active fluctuations we assume Gaussian noise acting with intensity $D_{v}$ along the heading direction. It is complemented by a second Gaussian noise randomly changing the heading direction with intensity $D_{\varphi}$, by acting only perpendicularly to the heading vector. Therefore, we define

$$
\boldsymbol{\eta}_{\mathrm{a}}(t)=\sqrt{2 D_{v}} \xi_{v}(t) \boldsymbol{e}_{h}(t)+\sqrt{2 D_{\varphi}} \xi_{\varphi}(t) \boldsymbol{e}_{\varphi}(t)
$$

Again both noise sources shall be independent

$$
\left\langle\xi_{i}(t)\right\rangle=0, \quad\left\langle\xi_{i}(t) \xi_{j}\left(t^{\prime}\right)\right\rangle=\delta_{i, j} \delta\left(t-t^{\prime}\right), \quad i, j \in\{v, \varphi\}
$$

A similar decomposition happens in case of active noise compared to the Cartesian formulation with passive noise. The dynamics along the heading direction with velocity component $v$ decouple from the angular motion. This stochastic $\varphi-$ dynamics with homogeneous additive noise along circles of constant $v$ results even in an equipartition along $\varphi$. Subsequently the $v$ dynamics can be solved. As a result, models with active fluctuations becomes a new class of analytically tractable systems. 
3.2 Energy depot

To explain measurements of the velocity distribution function of the Euglena cells, we will use the depot model as introduced by Ebeling, Schweitzer and Tilch [25, 4143. This model traces the propulsion back to a food uptake of the cells which rate $q$ from the environment. It fills up an internal energy depot $e_{i n}(t)$ of the cell. The internal energy decay with rate $c$ and can be transformed into kinetic energy with rate $d$, whereby the corresponding term is proportional to the internal and kinetic energy of the cell. Thus $e_{i n}(t)$ obeys

$$
\dot{e}_{i n}=q-c e_{i n}-d e_{i n} v^{2} .
$$

The gained kinetic energy is transformed into momentum and creates a propulsion in the Newtonian force balance along the heading direction as

$$
\dot{v}=d e(t) v(t)-\gamma v+\sqrt{2 D_{v}} \xi_{v}(t) .
$$

We assume that the angular dynamics are purely stochastic, driven by Gaussian white noise:

$$
\dot{\varphi}=\frac{1}{v} \sqrt{2 D_{\varphi}} \xi_{\varphi}(t)
$$

This assumption implies also that the angular dynamics are not affected by the depot. In general, this might be not the case, for example angular fluctuation could decrease with decreasing energy depot. However, this would only affect the persistence of the cell trajectory and would make no difference to stationary velocity distributions we focus on here. The active noise sources $\xi_{k}(t)$ are defined in Eq. 8 .

Assuming a fast relaxation rate of the depot variable, the internal energy responds instantaneously to its supply and demand. Thus it assumes a stationary value and can be adiabatically eliminated, despite ongoing exchange with the environment (for a further discussion including stochastic supply $q$, see [25]). It yields

$$
e(t)=\frac{q}{c+d v^{2}(t)},
$$

and in consequence the heading velocity changes as

$$
\dot{v}=\frac{d q}{c+d v^{2}} v-\gamma v+\sqrt{2 D_{v}} \xi_{v}(t)
$$

Hence, the propulsive acceleration reads in this approximation

$$
a_{p}=\frac{d q}{c+d v^{2}} v
$$

Finally, the depot model becomes

$$
\dot{v}=a_{p}-\gamma v+\sqrt{2 D_{v}} \xi_{v}(t), \quad \dot{\varphi}=\frac{1}{v} \sqrt{2 D_{\varphi}} \xi_{\varphi}(t) .
$$

and the corresponding Fokker-Planck equation

$$
\frac{\partial}{\partial t} p_{a}\left(v, \varphi, t \mid v_{0}, \varphi_{0}, t_{0}\right)=-\frac{\partial}{\partial v}\left(a_{p}-\gamma v\right) p_{a}+D_{v} \frac{\partial^{2}}{\partial v^{2}} p_{a}+\frac{D_{\varphi}}{v^{2}} \frac{\partial^{2}}{\partial \varphi^{2}} p_{a} .
$$


with $a_{p}$ from Eq. 16.

The propulsion dynamics can be reformulated by introducing the stationary propulsion speed in the absence of noise $v_{0}$ defined as

$$
v_{0}=\sqrt{\frac{q}{\gamma}-\frac{c}{d}}
$$

Eq. 15 becomes then

$$
\dot{v}=\frac{\gamma d}{c+d v^{2}} v\left(v_{0}^{2}-v^{2}\right)+\sqrt{2 D_{v}} \xi_{v}(t)
$$

In case that parameters obey $\frac{q}{\gamma}-\frac{c}{d} \geq 0, v_{0}$ exists and is a stable fixed point of the deterministic speed dynamics. Its positive root $v_{0} \geq 0$ is the mean propulsion speed in the small noise limit. The depot pumps sufficient energy to excite a stationary motion. In the opposite case, the single stationary stable solution of the deterministic part of Eq. (15) is the rest state $\left(v_{0}=0\right)$. The depot is insufficient to generate persistent motion at the given damping $\gamma$. Thus, in this case particles are driven by the noise alone. Note that this noise has to be still associated with active fluctuations produced by the propulsion mechanism of the algae as it acts only along the heading direction, which is fundamentally different from the case of thermal agitation.

Later on, we estimate the value of $v_{0}$ from Eq. (19) using experimentally fitted parameters.

\subsection{Additive active noise}

The asymptotic stationary distribution $\lim _{t_{0} \rightarrow-\infty} p_{a}\left(v, \varphi, t \mid v_{0}, \varphi_{0}, t_{0}\right)=p_{a}(v)$ becomes independent of the initial conditions. Also the noisy $v(t)$ dynamics become independent from the direction $\varphi$ and in the stationary limit the probability function density decomposes $p_{a}(v, \varphi)=p_{a}(v) p_{d}(\varphi)$. The angular distribution becomes homogeneous in all directions $\varphi$ with $p_{d}(\varphi)=1 / 2 \pi$. The remaining probability density function for the velocity follows from the stationary Fokker-Planck equation

$$
\frac{\partial}{\partial t} p_{a}(v)=0=-\frac{\partial}{\partial v}\left(a_{p}-\gamma v\right) p_{a}+D_{v} \frac{\partial^{2}}{\partial v^{2}} p_{a}
$$

with $a_{p}$ from Eq. (16). The latter ordinary differential equation can be integrated with vanishing probability flux. Thus, the stationary probability density function of velocities projected on the heading dynamics reads 1

$$
p_{a}(v) \propto\left(\frac{c}{d}+v^{2}\right)^{\frac{q}{2 D_{v}}} \exp \left(-\frac{1}{2 D_{v}} \gamma v^{2}\right) .
$$

From this, by summing the positive and negative projected velocities, we obtain the speed distribution, i.e. $\tilde{p}(s)=p_{a}(s=v)+p_{a}(s=-v){ }^{2}$ It reads

$$
\tilde{p}(s)=p_{a}(s=v)+p_{a}(s=-v) \propto\left(\frac{c}{d}+s^{2}\right)^{\frac{q}{2 D v}} \exp \left(-\frac{1}{2 D_{v}} \gamma s^{2}\right) .
$$

\footnotetext{
1 Probability density functions are defined up to the normalization constant.

2 Note, it follows from this definition that the derivative of the speed distribution $\tilde{p}(s)$ vanishes in the limit $s \rightarrow 0$ if $p_{a}$ is differentiable at $v=0$.
} 

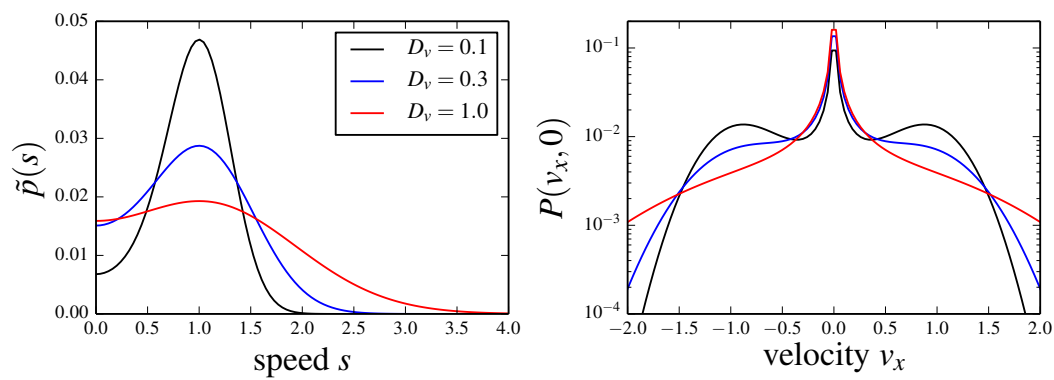

Fig. 5 The stationary speed probability (Eq. 23), left panel) and velocity distribution function (Eq. (24), right panel) for the energy depot with additive noise. Parameters: $q=2, d=1, c=$ $1, \gamma=1$ and noise intensity $D_{v}$ as assigned in the inset.

Transformation into the Cartesian velocities requires the consideration of the Jacobi-determinant for the two-dimensional variable transformation $s, \varphi \rightarrow v_{x}, v_{y}$. Therefore a giant peak occurs at the origin reflecting the non-vanishing speed probability density for the "resting" situation in the presence of active noise. Finally, we obtain

$$
P\left(v_{x}, v_{y}\right) \propto \frac{1}{\sqrt{v_{x}^{2}+v_{y}^{2}}}\left(\frac{c}{d}+v_{x}^{2}+v_{y}^{2}\right)^{\frac{q}{2 D v}} \exp \left(-\frac{1}{2 D_{v}} \gamma\left(v_{x}^{2}+v_{y}^{2}\right)\right) .
$$

The divergence of the Cartesian probability density is a universal property of active noise acting along the heading direction [25, 29. It is intrinsically connected to finite values of the speed probability density at the origin $\tilde{p}(s=0)>0$. This in turn, is a consequence of active noise stochastically exploring speed states infinitely close to the resting situation. These states are never reached in case of an ordinary Brownian particle, where the permanent stochastic impacts, independent in $x$ - and $y$-direction, always drive the speed dynamics away from the state $s=0$.

The solution Eq. (22) looks very similar to the stationary solution in the depot model as reported in 44. However it has to be emphasized that it describes a different physical situation. The solution in 44 describes the Cartesian velocity components as the results from a passive noise as given by Eq. (7). In the case discussed here the solution was obtained along the co-moving heading direction and Cartesian velocity components are due to Eq. (24).

As shown in a previous paper 45 the existence of the state with a maximum in the probability density function can act as a strong attractor in a situation with many particles and a velocity-alignment interaction between particles. In this situation the particles with active noise are collectively "trapped" in the resting state in contrast if passive noise is applied. The character of the phase transition between the ordered (running particles) state and disordered state changes from a second order to a first order transition due to the coexistence of the running and resting states. 


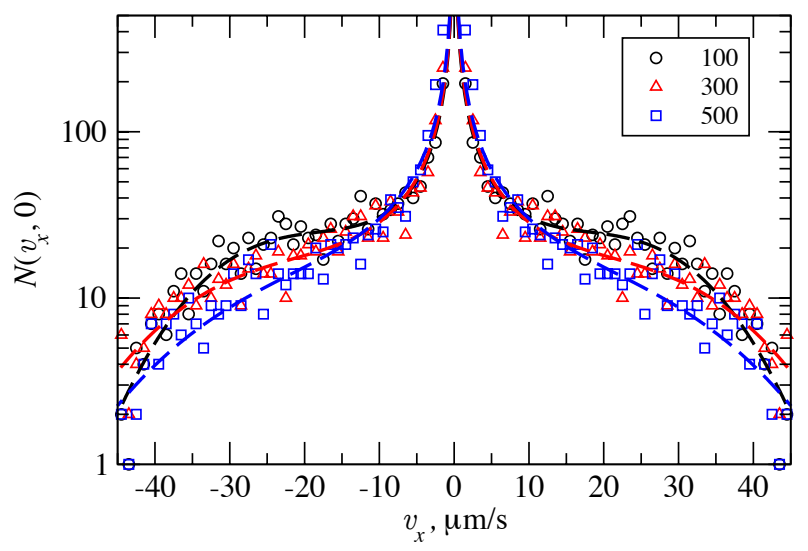

Fig. 6 Euglena gracilis one-dimensional velocity histograms collected in intense blue light (points) and the fits with Eq. 24 (lines). The labels on the experimental sets indicate the time interval within which the data were collected: 100 corresponds to the 0-100 seconds, 300 to 200-300 seconds, and 500 to 400-500 seconds from the beginning of the experiment.

\subsection{Comparison with measurements}

We now compare the observed Euglena cell velocity histograms with the predicted shape of the distribution. The histograms along with the theory curves are presented in Fig. 6. All the sets could be fitted equally well. We do not show all of them as they practically overlap. The correlation coefficient is in the range from 0.944 to 0.955 in different sets. We see that the cell motion matches very well the predicted shape for systems with active noise of high amplitude as shown in Fig. 5. It has a characteristic strong peak around zero velocity. The fit is good in the whole velocity range. Moreover, we observe that the distribution changes with time during the experiment. The peak close to zero velocity becomes even stronger with time while the wings of the distribution, corresponding to the normally moving cells, get somewhat weaker, which is consistent with the increase of the fluctuation amplitude $D_{v}$ (see curves with $D_{v}=0.3$ and 1.0 in Fig. 5 , right panel).

We attempted to interpret the latter observation using the depot model with active noise. The parameters of the motion are as shown in Table 1. As we see from the data, the ratio of energy influx to the fluctuation amplitude $q /\left(2 D_{v}\right)$ remains nearly constant during the experiment. The ratio $\gamma /(2 D v)$ is decreasing by about $50 \%$. Although we cannot resolve $D_{v}$ independently, we can assume that the viscous friction coefficient $\gamma$ stays constant as it reflects just the shape of the cells. We determined it in our previous work for Euglena cells $\gamma=1.2 \times 10^{-7}$ $\mathrm{N} /(\mathrm{m} / \mathrm{s})$ [46. Then, the decrease of the ratio $\gamma /\left(2 D_{v}\right)$ indicates the increase of the fluctuation amplitude by about $50 \%$. At the same time, as the ratio $q /\left(2 D_{v}\right)$ does not vary much (the variation is $5 \%-7 \%$ ), corresponding to values of $q=1.8 \times 10^{-16}$ $\mathrm{W}$ to $2.7 \times 10^{-16} \mathrm{~W}$, we can infer the increase of the incoming power by ca. $45 \%$. The ratio $c / d$ is increasing most significantly during the experiment, the total growth being more than $100 \%$. The propulsion velocity that is defined in the depot model [25, 41] by Eq. (19) stays fairly constant at first, $v_{0} \approx 26 \mu \mathrm{m} / \mathrm{s}$, but decreases to $18 \mu \mathrm{m} / \mathrm{s}$ towards the end. In the last interval the difference $q / \gamma-c / d$ turns negative and the propulsion velocity $v_{0}$ as parameter looses its meaning. The 


\begin{tabular}{ccccc} 
Time label & $q /\left(2 D_{v}\right)$ & $10^{-9} \gamma /\left(2 D_{v}\right)$ & $10^{9} c / d$ & $v_{0}, \mu \mathrm{m} / \mathrm{s}$ \\
\hline 100 & 11.2 & 5.6 & 1.3 & 25.9 \\
200 & 10.7 & 5.2 & 1.3 & 26.6 \\
300 & 10.7 & 3.8 & 2.1 & 26.9 \\
400 & 10.7 & 4.0 & 2.2 & 22.0 \\
500 & 10.5 & 3.3 & 2.9 & 17.7 \\
600 & 10.4 & 3.8 & 2.9 & $\mathrm{~N} / \mathrm{A}$ \\
\hline
\end{tabular}

Table 1 Parameters of the depot model with active fluctuations from fit of Eq. (24) to Euglena gracilis one-dimensional velocity histograms. There is no detectable persistent motion of the cells for the given damping $\gamma$ at time 500 to 600 seconds as $q / \gamma-c / d<0$.

second term $c / d$ expresses the rate of internal energy dissipation to energy transfer. Hence, the observation is consistent with the increase of internal dissipation in the cells during the experiment. The overall picture can be interpreted as follows: The Euglena cells exposed to intense illumination show negative phototaxis and are getting more and more fatigued at long exposures: The amplitude of velocity fluctuations as well are internal dissipation are growing while the fraction of energy that is converted into persistent motion is decreasing.

\subsection{Active multiplicative noise}

Here, we also demonstrate the application of the active noise concept to a model with multiplicative noise, which has been reported for random migration of the social amoeba Dictyostelium discoideum [8. The authors of this study measured the first two moments of the velocity increments per unit time as function of the velocity parallel and perpendicular to the direction of motion. From those data they approximated the propulsion function and the noise term of a corresponding Langevin equation.

In detail, they proposed based on their measurements, a simple linear friction term combined with a multiplicative noise term, with linear dependence on speed. Thus, the motion of the cells in their model is entirely driven by fluctuations without a deterministic component in the driving force. Please note that the authors considered in their model only passive noise, independent on the heading of the cell.

Here we go beyond the friction model suggested in [8] and calculate the speed distributions of self-propelled agents with active multiplicative noise and so-called Schienbein-Gruler friction function [25, 47]. The function includes a constant driving force along the heading direction

$$
\boldsymbol{a}_{p}(t)=\gamma v_{0} \boldsymbol{e}_{h}(t)
$$

and a linear damping by a Stokes friction $-\gamma v$ (c.f. Eq. 5). Thus for $v_{0}=0$ the model reduces to the situation observed in Dictyostelium migration [8]. However, for $v_{0}>0$, it also accounts for the case of individual agents with a finite preferred speed. Fluctuations along the heading direction depend on the velocity, whereas angular fluctuations are assumed to have constant intensity, i.e.

$$
\boldsymbol{\eta}_{\mathrm{a}}(t)=\sqrt{2 D_{v}} g(\boldsymbol{v}) \xi_{v}(t) \boldsymbol{e}_{h}+\sqrt{2 D_{\varphi}} \xi_{\varphi}(t) \boldsymbol{e}_{\varphi} .
$$



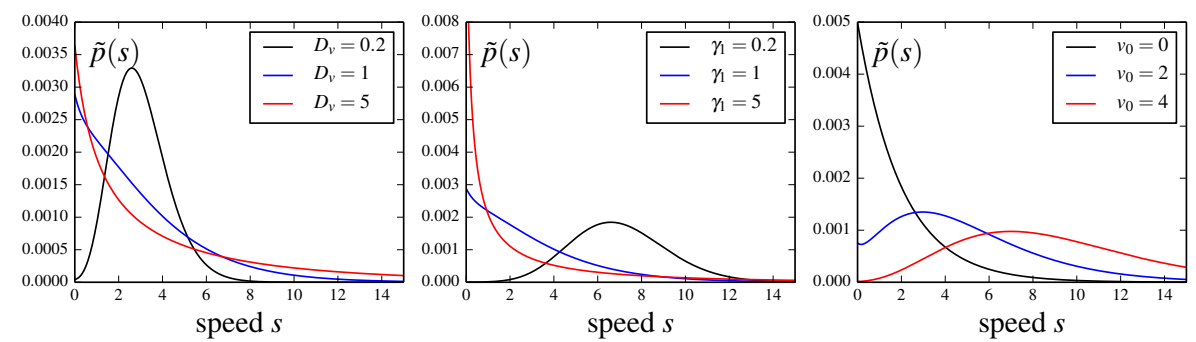

Fig. 7 Stationary speed distribution $\tilde{p}(s)$ Eq. 30 for the model with multiplicative noise Eq. 277 for different values of $D_{v}$ (left), $\gamma_{1}$ (center) and $v_{0}$ (right). Default parameters if not varied: $\gamma=1.0, \gamma_{1}=1.0, D_{v}=1.0, v_{0}=1.0$.

Here, $\xi_{i}(t)$ with $i, j=v, \varphi$ are again sources of independent Gaussian white noise defined in Eq. (8). In agreement with [8] we consider the case

$$
g(\boldsymbol{v})=\left(1+\gamma_{1}|v|\right) .
$$

and $v$ is the velocity component along the heading direction. Thus, the multiplicative noise grows along the heading direction in both directions. We would like to underline that the additive part in the noise for $v=0$ together with the Gaussian character of this noise can perform transitions from a motion with a positive velocity along the heading direction to a motion with negative one and vice versa.

Using the Stratonovich interpretation [48, we can now calculate the stationary speed distribution. The distribution $p_{a}\left(v, \varphi, t \mid v_{0}, \varphi_{0}, t_{0}\right)$ of the projected velocities $v$ along the heading direction and on its angle $\varphi$ obeys the Fokker-Planck equation

$$
\frac{\partial p_{a}}{\partial t}=-\frac{\partial}{\partial v} \gamma\left(v_{0}-v\right) p_{a}+D_{v} \frac{\partial}{\partial v}\left(1+\gamma_{1}|v|\right) \frac{\partial}{\partial v}\left(1+\gamma_{1}|v|\right) p_{a}+\frac{D_{\varphi}}{v^{2}} \frac{\partial^{2}}{\partial \varphi^{2}} p_{a} .
$$

The asymptotic stationary distribution $\lim _{t_{0} \rightarrow-\infty} p_{a}\left(v, \varphi, t \mid v_{0}, \varphi_{0}, t_{0}\right)=p_{a}(v)$ becomes independent on the initial conditions and the angle. With continuity of the velocity distribution function density $p_{a}(v)$ at the origin $v=0$ we obtain

$$
p_{a}(v) \propto\left(1+\gamma_{1}|v|\right)^{\left(-1+\frac{\gamma}{D v \gamma_{1}^{2}}+\frac{\gamma v_{0}}{D v \gamma_{1}} \frac{v}{|v|}\right)} \exp \left(-\frac{\gamma}{2 D_{v} \gamma_{1}}|v|\right) .
$$

Due to the lack of symmetry of the propulsion $v_{0}>0$, the probability density function in $p_{a}(v)$ is also asymmetric with respect to the origin $v=0$. Since the noise depends on the absolute value $|v|$, it is symmetric and grows in negative and as well positive direction. This dependence induces different slopes for negative and positive velocities around $v=0$ and $p_{a}$ is not differentiable at the origin for $v_{0}>0$.

Summation of probability at positive and negative velocities $v$ values yields the speed distribution

$$
\tilde{p}(s) \propto\left(1+\gamma_{1} s\right)^{\left(-1+\frac{\gamma}{D_{v} \gamma_{1}^{2}}\right)} \exp \left(-\frac{\gamma}{2 D_{v} \gamma_{1}} s\right)\left(\left(1+\gamma_{1} s\right)^{\left(\frac{\gamma v_{0}}{D v \gamma_{1}}\right)}+\left(1+\gamma_{1} s\right)^{-\left(\frac{\gamma v_{0}}{D v \gamma_{1}}\right)}\right)
$$



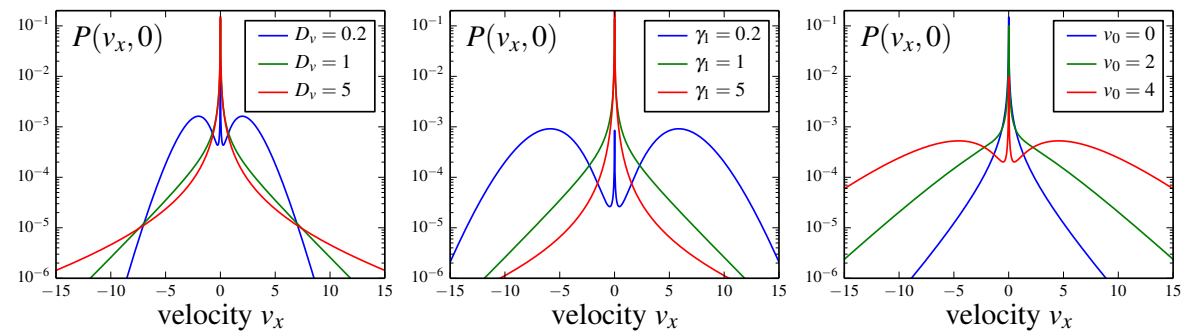

Fig. 8 Stationary velocity distribution of the $v_{x}$-velocity component $P\left(v_{x}, 0\right)$, Eq. 31 for the model with multiplicative noise Eq. 27) for different values of $D_{v}$ (left), $\gamma_{1}$ (center) and $v_{0}$ (right). Default parameters if not varied: $\gamma=1.0, \gamma_{1}=1.0, D_{v}=1.0, v_{0}=1.0$.

Interestingly, the asymptotic behavior for large speed values decays exponentially and not as a Gaussian. In Fig. 7 we show examples of the speed distribution given in Eq. (30) for different parameter sets.

Finally, we transform the above results to Cartesian coordinates and obtain the two-dimensional Cartesian velocity probability density, which diverges at the origin:

$$
P\left(v_{x}, v_{y}\right)=\frac{1}{\sqrt{v_{x}^{2}+v_{y}^{2}}} \tilde{p}\left(s=\sqrt{v_{x}^{2}+v_{y}^{2}}\right)
$$

The corresponding graphs of this function for $v_{y}=0$ are shown in Fig. 8 for different values of $D_{v}, \gamma_{1}$ and $v_{0}$ (as in Fig. 7). We stress that as in the case of additive noise, we obtain a pronounced peak of the velocity distribution at vanishing velocities.

\section{Conclusions}

In this work, we have discussed the concept of self-propelled particles with active fluctuations in the context of experimentally measured velocity distributions of Euglena gracilis algae. In biological agents, the locomotion is controlled by multiple internal decision processes, which cannot be easily accessed. These complex, unresolved influences will strongly contribute to the apparent stochasticity of the motion, and in the first approximation they can be conveniently summarized as active fluctuations. The intensity of such active fluctuations and its dependence on various environmental parameters yield important information on the internal states of the biological agents and determines ecologically relevant quantities as for example the spatial dispersal (diffusion) of individuals [49. Here, our results show that the depot model [41] of active Brownian motion together with active additive fluctuations perfectly describes experimentally measured velocity distributions. The fitting of theoretical distributions to experimental data reveals a systematic change of locomotion parameters of individual algae with the duration of the experiment, which provides important insights into the adaptation of individual cells to a stressful environment.

We should add that the Euglena single-cell algae might demonstrate a more complicated behavior as it was reported previously. It might be one of the simplest 
systems showing fatigue in response to external stress. Although we successfully described the population statistics, on the single cell level the mechanism of the active fluctuations and of the observed "fatigue" remains unclear. This mechanism can be investigated further if one addresses complex and not fully understood impulse-response behaviors involving the photoactivated adenylyl cyclase [39, 50] and protein kinase [51].

The theory of active fluctuations predicts a divergence of two-dimensional Cartesian velocity probability densities irrespective on the propulsion details [29]. The corresponding diverging probability density manifests itself by a finite (local) maximum at the origin, as it is the case in our experimental data. Similar maxima in Cartesian distributions have been observed also in very different experimental systems such as migrating Dictyostelium discoideum amoeba [9] or self-propelled colloids [52]. Thus we expect that active fluctuations are ubiquitous in living and non-living active matter systems and therefore should be taken into account in interpretation of corresponding experimental results and their theoretical description.

\section{Acknowledgements}

This work has been supported by the Deutsche Forschungsgemeinschaft via IRTG 1740 (L. S.-G.). P. R. acknowledges support by the DAAD via the P.R.I.M.E. fellowship.

\section{References}

1. A. Einstein, Ann. Phys. 17(8), 549 (1905)

2. P. Langevin, C. R. Hebd. Seances Acad. Sci 146, 530 (1908)

3. M. von Smoluchowski, Ann. Phys. 326(14), 756 (1906)

4. P. Reimann, Phys. Rep. 361(2-4), 57 (2002)

5. B.M. Friedrich, F. Jülicher, Proc. Natl. Acad. Sci. USA 104(33), 13256 (2007)

6. S. van Teeffelen, H. Löwen, Phys. Rev. E 78(2), 020101 (2008)

7. D. Selmeczi, L. Li, L.I. Pedersen, S.F. Nørrelykke, P.H. Hagedorn, S. Mosler, N.B. Larsen, E.C. Cox, H. Flyvbjerg, Eur. Phys. J. Special Topics 157(1), 1 (2008)

8. H. Bödeker, C. Beta, T. Frank, E. Bodenschatz, Europhys. Lett. 90(2) (2010)

9. L. Li, S.F. Nørrelykke, E.C. Cox, PLoS ONE 3(5), e2093 (2008)

10. R. Dilao, M.J.B. Hauser, Comptes Rendus Biologies 336(11-12), 565 (2013)

11. H. Niwa, J. Theor. Biol. 171(2), 123 (1994)

12. N. Komin, U. Erdmann, L. Schimansky-Geier, Fluct. Noise Lett. 4(1), L151 (2004)

13. S. Bazazi, P. Romanczuk, S. Thomas, L. Schimansky-Geier, J.J. Hale, G.A. Miller, G.A. Sword, S.J. Simpson, I.D. Couzin, Proc. R. Soc. B: Biol. Sci. (2010)

14. W.F. Paxton, K.C. Kistler, C.C. Olmeda, A. Sen, S.K.S. Angelo, Y. Cao, T.E. Mallouk, P.E. Lammert, V.H. Crespi, J. Am. Chem. Soc. 126(41), 13424 (2004) 
15. J.R. Howse, R.A.L. Jones, A.J. Ryan, T. Gough, R. Vafabakhsh, R. Golestanian, Phys. Rev. Lett. 99(4), 048102 (2007)

16. G. Ruckner, R. Kapral, Phys. Rev. Lett. 98(15), 150603 (2007)

17. K.V. Kumar, S. Ramaswamy, M. Rao, Phys. Rev. E 77(2), 020102 (2008)

18. P. Tierno, R. Albalat, F. Sagués, Small 6(16), 1749 (2010)

19. T. Vicsek, A. Czirók, E. Ben-Jacob, I. Cohen, O. Shochet, Phys. Rev. Lett. 75(6), 1226 (1995)

20. I.D. Couzin, J. Krause, R. J., G.D. Ruxton, N.R. Franks, J. Theor. Biol. 218(1), 1 (2002)

21. H. Chate, F. Ginelli, R. Montagne, Phys. Rev. Lett. 96(18), 180602 (2006)

22. J. Buhl, D.J.T. Sumpter, I.D. Couzin, J.J. Hale, E. Despland, E.R. Miller, S.J. Simpson, Science 312(5778), 1402 (2006)

23. A. Sokolov, I.S. Aranson, J.O. Kessler, R.E. Goldstein, Phys. Rev. Lett. 98(15), $158102(2007)$

24. A. Baskaran, M.C. Marchetti, Proc. Natl. Acad. Sci. USA 106(37), 15567 (2009)

25. P. Romanczuk, M. Bär, W. Ebeling, B. Lindner, L. Schimansky-Geier, Eur. Phys. J. Special Topics 202, 1 (2012)

26. M.C. Marchetti, J.F. Joanny, S. Ramaswamy, T.B. Liverpool, J. Prost, M. Rao, R.A. Simha, Rev. Mod. Phys. 85, 1143 (2013)

27. A. Kudrolli, G. Lumay, D. Volfson, L.S. Tsimring, Phys. Rev. Lett. 100(5), $058001(2008)$

28. F. Peruani, L.G. Morelli, Phys. Rev. Lett. 99(1), 010602 (2007)

29. P. Romanczuk, L. Schimansky-Geier, Phys. Rev. Lett. 106, 230601 (2011)

30. M. Theves, J. Taktikos, V. Zaburdaev, H. Stark, C. Beta, Biophys. J. 105, 1915 (2013)

31. F. Detcheverry, Eur. Phys. J. E 37, 114 (2014)

32. F. Thiel, L. Schimansky-Geier, I.M. Sokolov, Phys. Rev. E 86, 021117 (2012)

33. D. Mizuno, C. Tardin, C.F. Schmidt, F.C. MacKintosh, Science 315(5810), 370 (2007)

34. C.P. Brangwynne, G.H. Koenderink, F.C. MacKintosh, D.A. Weitz, Trends in Cell Biology 19(9), 423 (2009)

35. E. Fodor, K. Kanazawa, H. Hayakawa, P. Visco, F. van Wijland, Phys. Rev. E 90(4), 042724 (2014)

36. R. Ma, G.S. Klindt, I.H. Riedel-Kruse, F. Jülicher, B.M. Friedrich, Phys. Rev. Lett. 113(4), 048101 (2014)

37. B. Deihn, Biochim. Biophys. Acta 177, 136 (1969)

38. K. Ozasa, J. Lee, S. Song, M. Maeda, Plant Cell Physiol. 55(10), 1704 (2014)

39. M. Ntefidou, M. Iseki, M. Watanabe, M. Lebert, D. Häder, Plant Physiol. 133, $1517(2003)$

40. K. Ozasa, L. Lee, S. Song, M. Hara, M. Maeda, Appl. Soft Comp. 13, 527 (2013)

41. F. Schweitzer, W. Ebeling, B. Tilch, Phys. Rev. Lett. 80, 5044 (1998)

42. W. Ebeling, F. Schweitzer, B. Tilch, Biosystems 49, 17 (1999)

43. F. Schweitzer, Brownian Agents and Active Particles: Collective Dynamics in the Natural and Social Sciences. Synergetics (Springer, 2003)

44. U. Erdmann, W. Ebeling, L. Schimansky-Geier, F. Schweitzer, Eur. Phys. J. B 15, 105 (2000) 
45. R. Großmann, L. Schimansky-Geier, P. Romanczuk, New J. Phys. 14(7), 073033 (2012)

46. M. Romensky, D. Scholz, V. Lobaskin, Interface 99, 99 (2014)

47. M. Schienbein, H. Gruler, Bull.Math.Biol. 55, 585 (1993)

48. V. Anishchenko, T. Astakhov, Vadivasova, A. Neiman, L. Schimansky-Geier, Nonlinear Dynamics of Chaotic and Stochastic Systems. Synergetics (Springer, 2003)

49. P. Romanczuk, Active Motion and Swarming. From Individual to Collective Dynamics, Nichtlineare und Stochastische Physik, vol. 12 (Logos Verlag, Berlin, 2011)

50. M. Ntefidou, D. Häder, Photochem. Photobiol. Sci. 4(9), 732 (2005)

51. V. Daiker, D. Häder, P. Richter, M. Lebert, Planta 233(5), 1055 (2011)

52. H. Ke, S. Ye, R.L. Carroll, K. Showalter, J. Phys. Chem. A 114(17), 5462 (2010) 